\title{
Arquitetura Sísmica de Borda de Plataforma como um Diagnóstico de Variações Eustáticas Pleistocênicas na Porção Sul da Bacia de Campos
}

Luís Vinícius Jordão Teixeira 1*, Antonio Tadeu dos Reis 2, Cleverson Guizan Silva 3, Renata Moreira da Costa Maia 4. Rio de Janeiro, RJ - Faculdade Federal Fluminense - UFF.

Copyright 2014, SBGf - Sociedade Brasileira de Geofísica.

Este texto foi preparado para a apresentação no VI Simpósio Brasileiro de Geofísica, Porto Alegre, 14 a 16 de outubro de 2014. Seu conteúdo foi revisado pelo Comitê Técnico do VI SimBGf, mas não necessariamente representa a opinião da SBGf ou de seus associados. É proibida a reprodução total ou parcial deste material para propósitos comerciais sem prévia autorização da SBGf.

\section{Resumo}

Foi identificado na porção sul da bacia de Campos um conjunto de 10 subunidades sísmicas distintas compondo os primeiros $300 \mathrm{~ms}$ de profundidade do arcabouço deposicional (sequências Sq3 e Sq4 identificadas por Marangoni, 2012). Foi realizada uma estimativa da acomodação sedimentar desenvolvida durante a formação das subunidades na região de borda de plataforma com a intenção de associá-la com os ciclos de $4^{\mathrm{a}}-5^{\mathrm{a}}$ ordem que compões as grandes janelas temporais referentes aos ciclos de Milankovitch, tornando possível estimar idades de formação para as subunidades deposicionais.

\section{Abstract}

A set of 10 distinct seismic subunits composing the first $300 \mathrm{~ms}$ of depth of the depositional framework was identified in the southern portion of the Campos Basin (Sq3 and Sq4 sequences identified by Marangoni, 2012) were identified in the southern portion of the Campos Basin. An estimate of sediment accommodation developed during the formation of the subunits in the region of the edge of the platform with the intention of associating it with the cycles of 4th and 5th order which compose the great storms related to Milankovitch cycles windows was performed, making it possible to estimate ages training for depositional subunits.

\section{Introdução}

Em uma bacia, os parâmetros essenciais de controle da arquitetura estrutural da sucessão estratigráfica das margens são o tectonismo, a eustasia e o aporte sedimentar (Posamentier et al., 1993). A relação entre os movimentos verticais e a eustasia determinará o espaço de acomodação sedimentar de uma bacia, definido como o espaço a ser ocupado pelos sedimentos a partir de um determinado datum fixo (Jervey, 1988). A relação entre o espaço de acomodação sedimentar e a taxa de aporte sedimentar produzirá as diferentes geometrias possíveis de empilhamento sedimentar na região de borda de plataforma, como unidades tabulares de cobertura (draping units), primas de progradação frontal e/ou fácies onlapantes (Lobo et al., 2005). Marangoni (2012) identificou na porção sul da bacia de Campos um conjunto de quatro sequências deposicionais dominantemente progradacionais em direção à bacia, denominadas de Sq1-Sq4. Essas sequências são limitadas por superfícies bastante irregulares (superfícies
S1 a S5) sugerindo processos erosivos relacionados a longos períodos de exposição subaérea da plataforma continental, durante a queda relativa do nível do mar.

O presente trabalho tem por objetivos: (i) realizar uma releitura das sequências sísmicas Sq3 e Sq4 identificadas por Marangoni (2012) com o intuito de caracterizar as geometrias externas e as fácies sísmicas internas das subunidades; (ii) o que estas feições podem indicar em termos de variações locais de espaço de acomodação e aporte sedimentar entre os diferentes setores da plataforma estudada; e (iii) propor um modelo de deposição de trato de sistemas para as sequências Sq3 e Sq4, na tentativa de reconstruir a história deposicional da área e o impacto das variações climáticas do Quaternário Superior no registro sedimentar da plataforma.

\section{Base de dados e Metodologia}

Este estudo dispõe de cerca de $300 \mathrm{~km}$ de perfis de sísmica de reflexão monocanal coletados com sistema Sparker modelo SQUID 2000, Applied Acoustics Engeneering Ltda (potência de $700 \mathrm{~J}$ e penetração do sinal acústico de cerca de 250-300 ms), durante a Missão Oceanográfica Rio Costa II (2011), realizada em parceria com a Marinha do Brasil (Figura 1).

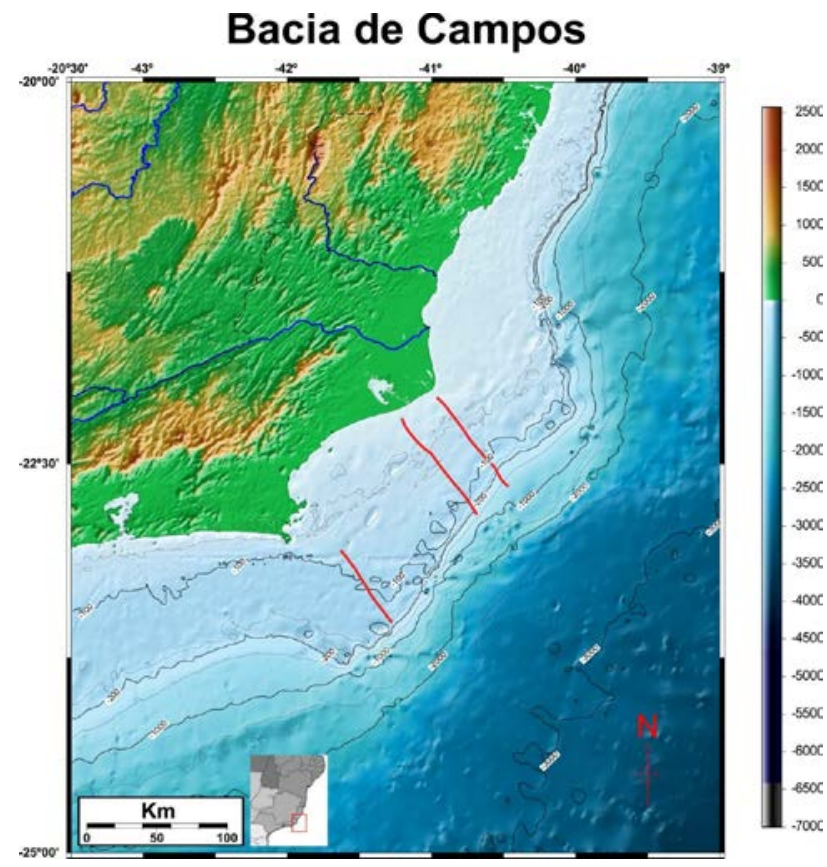

Figura 1: Mapa de localização da área de estudo, e da base de dados sísmicos e cronoestratigráficos utilizados neste trabalho. 
Os dados sísmicos adquiridos foram processados no próprio sistema de aquisição sísmica, modelo CODA 500, através da aplicação de filtros para melhoria da razão sinal/ruído e de filtros do tipo swell filter, para retirada dos ruídos de ondas do mar.

A interpretação dos perfis sísmicos se baseou nos princípios fundamentais da estratigrafia sísmica e de sequências de alta resolução (Mitchum et al., 1977; Catuneanu, 2006) e na sua comparação com estudos de caso em escala mundial (Tesson et al., 1990; Mitchum e Van Wagoner, 1991; Evans et al., 1995; Foyle e Oertel, 1997; Hernandez-Molina et al., 2000; Muto e Steel, 2000; Tesson et al., 2000; Catuneanu, 2002; Jin et al., 2002; Ridente e Trincardi 2002; Trincardi e Correggiari, 2002; Cattaneo e Steel, 2003; Lofi et al., 2003; Catuneanu, 2006; Rabineau et al., 2006; Tripsanas e Piper, 2008).

\section{Resultados}

As sequências sedimentares Sq3-Sq4 identificadas por Marangoni (2012) e reinterpretadas neste trabalho são limitadas pelas superfícies S3, S4 e S5, e foram subdivididas, respectivamente, em seis e quatro subunidades deposicionais distintas (Sq3: Sb_A, Sb_B, Sb_C, Sb_D, Sb_E, e Sb_F, e Sq4: Sb_G, Sb__H, Sb̄_I, $\mathrm{Sb} \_$J).

Na porção sul da área de estudo (adjacente à Cabo Frio) as subunidades Sb_A, Sb_C e Sb_E assentam-se sobre discordâncias erosivas, na forma de lençol com fácies constituídas por refletores internos subparalelos e descontínuos, variando entre $2-8 \mathrm{~m}$ de espessura ao longo da plataforma continental (Figura 2).

As subunidades Sb_B e Sb_D são caracterizadas por complexo de clinoformas oblíquas sigmoidais que gradam no sentido plataforma interna-externa para clinoformas oblíquas paralelas numa relação de baixo ângulo com a superfície topo, bastante irregular e erodida, semelhante a sistemas praias com foreset migrando no sentido da progradação e offlap descendente (Figura 2).

A subunidade $\mathrm{Sb} F$ apresenta-se como um prisma de borda de plataforma que se espessa na direção da progradação e é erodido por escalonamentos e canais. Sua espessura varia de $\sim 6-30 \mathrm{~m}$ ao longo da plataforma continental e $\sim 80 \mathrm{~m}$ no talude superior (Figura 2).

A subunidade Sb_G e Sb_I é caracterizada por geometria externa tabular, com refletores internos subparalelos e descontínuos compondo um sistema de preenchimento fluvio-estuarino e depressões erosivas preservadas ao longo da plataforma continental. A subunidade Sb_G recobre cerca de $-35 \mathrm{~km}$ da plataforma continental sul da área com espessuras em torno de -2-9 m (Figura 2).

A subunidade $\mathrm{Sb} \mathrm{H}$ é composta basicamente por clinoformas oblíquas de baixo ângulo em direção à bacia. A subunidade mostra-se entalhada por canais e depressões erosivas em regiões mais internas da área. Escarpas maiores ( $-2-3 \mathrm{~km}$ de comprimento e $\sim 8 \mathrm{~m}$ de altura) individualizam patamares morfológicos em direção à borda de plataforma (Figura 2).
A Subunidade Sb_J é caracterizada por geometria prismática e clinoformas oblíquas sigmoidais gradando para clinoformas oblíquas paralelas ao longo da plataforma continental. Apresenta-se erodida por truncamentos erosivos e escarpas que individualizam patamares erosivos com desníveis morfológicos na ordem de $\sim 7 \mathrm{~m}$ de altura em profundidades de $\sim-90 \mathrm{~m}$. A subunidade avança em direção à quebra de plataforma com espessuras que variam entre $\sim 15 \mathrm{~m}$ até $40 \mathrm{~m}$ na borda de plataforma (Figura 2).

\section{Cálculo do Espaço de Acomodação e Subsidência}

Para a estimativa da acomodação sedimentar desenvolvida durante a formação das subunidades foi utilizada a curva eustática baseada nos dados isotópicos de Oxigênio $\delta 18$ propostas por Bassinot et al. (1994) e Lisiecki e Raymo (2005) (Figura 3) e a taxa de subsidência calculada sobre os perfis sísmicos utilizados.

Considerando as superfícies limitantes das sequências Sq3 e Sq4 como descontinuidades erosivas, foi possível estimar a taxa de subsidência durante os respectivos períodos de deposição relacionando os respectivos offlap breaks com níveis de mar mais baixos referentes aos estágios isotópicos MIS2, MIS6 e MIS8 (resumidos na tabela da Figura 4). Seguindo o modelo proposto por Rabineau et al. (2013), a medida entre o offlap break da superfície base de uma sequência e a linha tangente à plataforma da sua superfície topo, indica o quanto de espaço de acomodação (subsidência + variação eustática) foi criado durante a deposição de cada sequência. A variação eustática entre os períodos de formação dos offlap breaks das superfícies estratigráficas S4 e S5, relacionados aos respectivos estágios isotópicos MIS6 e MIS8, indica o quanto houve de destruição/criação de espaço de acomodação durante a formação de cada sequencia. A partir dos valores de espaço de acomodação e de variação eustática foi possível, então, estimar as taxas de subsidência de cada uma das sequências $\mathrm{Sq} 3$ e $\mathrm{Sq} 4$ e as idades de formação das subunidades $\mathrm{Sb} D, \mathrm{Sb} F, \mathrm{Sb} H$ e Sb_J através da mesma metodologia (valores resumidos na tabela da Figura 4).

\section{Discussão e Conclusões}

A seção sísmica interpretada a sul da área (Linha 16) mostra na região de borda de plataforma um perfil retrogradante refletindo o recuo do offlap break em direção ao continente, apesar do processo de agradação ser visivelmente ocorrente. As variações na espessura, no grau de preservação dos elementos arquiteturais e alcance interno das subunidades sísmicas acusam acomodação diferencial (destruição/criação de espaço de acomodação) ao longo da plataforma continental durante os ciclos de exposição subaérea (Figura 2). Esta variação pode ser de natureza flexural ou por compactação, sob influência da descarga do rio Paraíba do Sul, mas ainda não é determinada.

A integração dos resultados da análise sísmica com as curvas eustáticas de dados isotópicos de $\delta 180$ (Rabineau et al., 2006) indicou que as sequências Sq3 e Sq4, empilhadas sobre a superfície S3, depositaram-se 
num intervalo temporal de cerca de $\sim 250 \mathrm{ka}$, sendo possível correlacionar as duas unidades sísmicas com subunidades deposicionais desenvolvidas durante subciclos glacio-eustáticos de mais alta frequência $\left(4^{\mathrm{a}}-5^{\mathrm{a}}\right.$ ordem) com cerca de 20-40 ka de duração e $\sim 70-80$ m de amplitude. Na bacia de Campos, a sequência Sq3 apresenta maior espessura em toda a plataforma continental, embora seu ciclo construcional reflita queda de nível de base de -118 $\mathrm{m}$, semelhante à sequência Sq4, com - $120 \mathrm{~m}$, inferindo que a taxa de criação de espaço de acomodação durante sua deposição (Sq3) tenha sido maior. A sequência $\mathrm{Sq} 4$ mostra-se menos espessa, refletindo o impacto na arquitetura sedimentar associado a ciclos de menor ordem dentro do grande ciclo de $\sim 100-120 \mathrm{ka}$, como mostra a curva de variação do nível do mar (Figura 3).

Os subciclos eustáticos de menor ordem são responsáveis por compor o principal controle nos ciclos deposicionais e padrões de acamamento interno das plataformas continentais (ex., Ridente e Trincardi, 2002; Rabineau et al., 2006; Tripsanas \& Piper, 2008). O resultado para a porção sul da bacia de Campos foi o empilhamento, ao longo da plataforma continental, de um conjunto de prismas sedimentares preservados sob regimes de queda do nível relativo de mar e exposição subaérea da plataforma continental à erosão desenvolvida sob condições de regressão forçada (as subunidades Sb_B, Sb_D, Sb_F, Sb_H e Sb_J). Preservados sob as superfícies de exposição subaérea encontram-se sistemas transgressivos de lençóis tabulares e preenchimento agradacional de canais fluvioestuarinos que recobrem quase que completamente a plataforma regressiva. Esta preservação está associada a maiores amplitudes e criação de espaço de acomodação durante as elevações eustáticas de menor ordem e mais alta frequência (as subunidades $\mathrm{Sb} A$, $\mathrm{Sb}$ C, Sb_E, Sb_G e Sb_l) dentro dos ciclos de $4^{-{ }^{a}} 5^{\mathrm{a}}$ ordem que compões as grandes janelas temporais referentes aos ciclos de Milankovitch.

O cruzamento da base de dados sísmicos, das informações da curva eustática de isótopos de Oxigênio $\delta 18$ e da acomodação estimada no período de formação de cada uma das subunidades deposicionais, forneceu as possíveis idades para as superfícies topo das subunidades encontradas Sb_D (MIS 7d), Sb_F (MIS 6) e Sb_J (MIS 2).

\section{Referências}

Bassinot, F., Labeyrie, L., Vincent, E., Quidelleur, X., Lancelot, N.J., Lancelot, 891 Y., 1994. The astronomical theory of climate and the age of the Brunhes - 892 Matuyama magnetic reversal, Earth Planet. Sci. Lett. 126. 91-108.

Cattaneo, A., Steel, R.J., 2003. Transgressive deposits: a review of their variability. Earth-Science Reviews 62, 187-228.

Catuneanu, O., 2002. Sequence stratigraphy of clastic systems: concepts, merits, and pitfalls. Journal of African Earth Sciences 35, 1-43.
Catuneanu, O. 2006. Principles of sequence stratigraphy. Elsevier: Oxford., 375 p.

Evans, C. D. R.; Brett, C. P.; James, J. W. C.; Holmes, R. Shallow seismic reflection profiles from the waters of east and southeast Asia - an interpretation manual and atlas. London: British Geological Survey, 1995. WC/94/60. Technical Report.

Foyle, A. M.; Oertel, G. F. Transgressive systems tract development and incised valley fills within a Quaternary estuary-shelf system: Virginia inner shelf, USA. Marine Geology, v. 137, p. 227-249. 1997.

Hernandez-Molina, F. J.; Somoza, L.; Lobo, F. Seismic stratigraphy of the Gulf of Cadiz continental shelf: a model for late Quaternary high resolution. In: Hunt, D.; Gaythorpe, R. (Eds.). Seismic response to forced regression. London: Geological Society Special Publication, 2000. v. 172, p. 329-362.

Jervey, M.T., 1988. Quantitative geological modeling of siliciclastic rock sequences and their seismic expression.

Jin, J. H.; Chough, S. K.; Ryang, W. H. Sequence aggradation and systems tracts partitioning in the mideastern Yellow Sea: roles of glacio-eustasy, subsidence and tidal dynamics. Marine Geology, v. 184, p. 249-271. 2002.

Lisiecki, L.E., Raymo, M.E., 2005. A PliocenePleistocene stack of 57 globally distributed benthic $\delta 18 \mathrm{O}$ records: Pliocene-Pleistocene Benthic Stack. Paleoceanography 20.

Lobo, F.J.; DIAS J.M.A.I.; Hernandez-Molina, F. J.; Gonzales, R., Fernandez-Salas, L.M. \& Diaz Del Rio, V. 2005. Late Quaternary shelf-margin wedges and upper slope progradation in the Gulf of Cadiz margin (SW Iberian Peninsula). CIACOMAR-CIMA, Universidade do Algarve, Olhão, Portugal. 8700-311.

Lofi, J.; Rabineau, M.; Gorini, C.; Berné, S.; Clauson, G.; Clarens, P.; Reis, A. T.; Mauntain, G. S.; Ryan, W. B. F.; Steckler, M. S.; Fauchet, C. Plioquaternary prograding clinoform wedges of the western gulf of lions continentalmargin (NW Mediterranean) after the messinian salinity crisis. Marine geology, v. 198, p. 3-4, p. 289-317. 2003.

Mariana B. Marangoni; Antonio Tadeu dos Reis; Cleverson G. Silva; Renata M. da Costa Maia; Josefa V. Guerra; Rodrigo A. Oliveira; Mayara M. Passos; Gabriela de A. Bernardo. 2012. Arcabouço Estratigráfico e Ciclicidade Deposicional dos Sistemas Sedimentares Quaternários da Plataforma Sul da Bacia de Campos (RJ). Universidade do Estado do Rio de Janeiro.

Mitchum Jr., R. M.; Vail, P. R.; Sangree, J. B. 1977. Seismic Stratigraphy and Global Changes of Sea Level, Part 6: Stratigraphy Interpretation of Seismic Reflection Patterns in Deposicional Sequences. In: C. E. Payton (ed.) Seismic Stratigraphy - Applications to Hydrocarbon Exploration. Tulsa, AAPG, pp. 294-312 (Memoir \#26). 
Mitchum, R.M., Van Wagoner, J.C., 1991. Highfrequency sequences and their stacking patterns: sequence-stratigraphic evidence of high-frequency eustatic cycles. Sedimentary Geology 70, 131-160.

Muto T \& Steel RJ. 2000. The accommodation concept in sequence.

Posamentier, H.W., Summerhayes, C.P., Haq, B.U., Allen, G.P., 1993. Sequence stratigraphy and facies associations. International Association of Sedimentology Special publication 18.

Rabineau, M.; Berné S.; Aslanian, D.; Olivet, J-L.; Joseph, P.; Guillocheau, F.; Bourillet , J-F.; Ledrezen, E.; Granjeon, D. 2006. Paleo sea levels reconsidered from direct observation of paleoshoreline position during Glacial Maxima (for the last 500,000 yr) Earth and Planetary Science Letters, 252, p. 119-137.

Rabineau, M., Leroux E., Aslanian, D., Bache, F., Gorini, C., Moulin, M., Molliex, S., Droz, L., Dos Reis, T., Rubino, J.L., Guillocheau, Olivet J.L. (in press). Quantifying subsidence and isostatic readjustment using sedimentary paleomarkers, example from the Gulf of Lion. Earth and Planetary Science Letters. DOI; 10.1016/j.epsl.2013.11.059.

Ridente, D. \& Trincardi, F. 2002. Eustatic and tectonic control on deposition and lateral variabily Quaternary regressive sequences in the Adriatic basin (Italy). Marine Geology, 184:273-293.

Tesson, M.; Ravene, C.; Allen, G.P. 1990. Applications des concepts de stratigraphie 186 séquentielle à un profil sismique haute résolution traverse à la plate-forme rhôdanienne. 187 Comptes Rendus de l'Academie de Sciences de Paris., 310(II):565- 570.

Tesson, M..; Posamentier, H. W.; Gensous, B. Stratigraphic organization of late Pleistecene deposits of the Western part of the Golfe du Lion shelf (Languedoc shelf) Western Mediterranea Sea, using high-resolution seismic and core data. AAPG Bulletin, v. 84, p.119-150. 2000.

Trincardi, F. \& Corregiari, A. 2000. Quaternary forcedregression deposits in the Adriatic basin and the record of composite sea-level cycles. In: Seismic response to forced regression. In: HUNT, D.; GAWTHORPE, R. L. (eds). Sedimentary responses to forced regressions. Geological Society, London, Special Publications, v. 172, p. 245-269.

Tripsanas, E. K. \& Piper, D. J.W. 2008. Late Quaternary stratigraphy and sedimentology of Orphan Basin: Implications for meltwater dispersal in the southern Labrador Sea Palaeogeography, Palaeoclimatology, Palaeoecology, 260(3-4):521-539. 

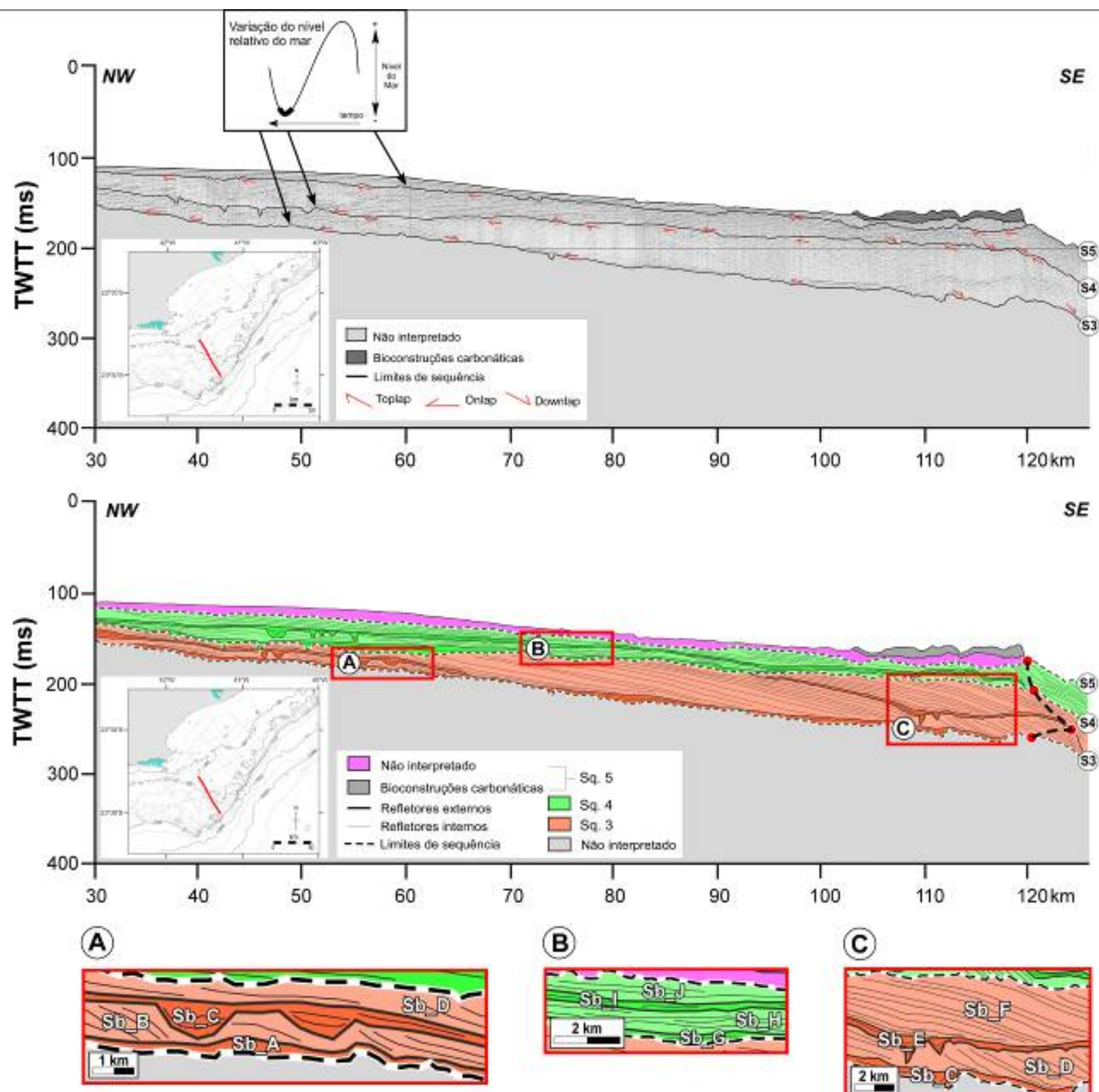

Figura 2: Perfil sísmico com orientação "dip" da base de dados Rio Costa 2, localizado na porção sul da Bacia de Campos e linedrawing interpretativo destacando os principais elementos arquiteturais e subunidades sísmicas das sequências Sq3-Sq4 identificados na área. Pontos em vermelho marcam a posição do offlap break em cada subunidade sísmica e pontilhado preto a trajetória do offlap break. 


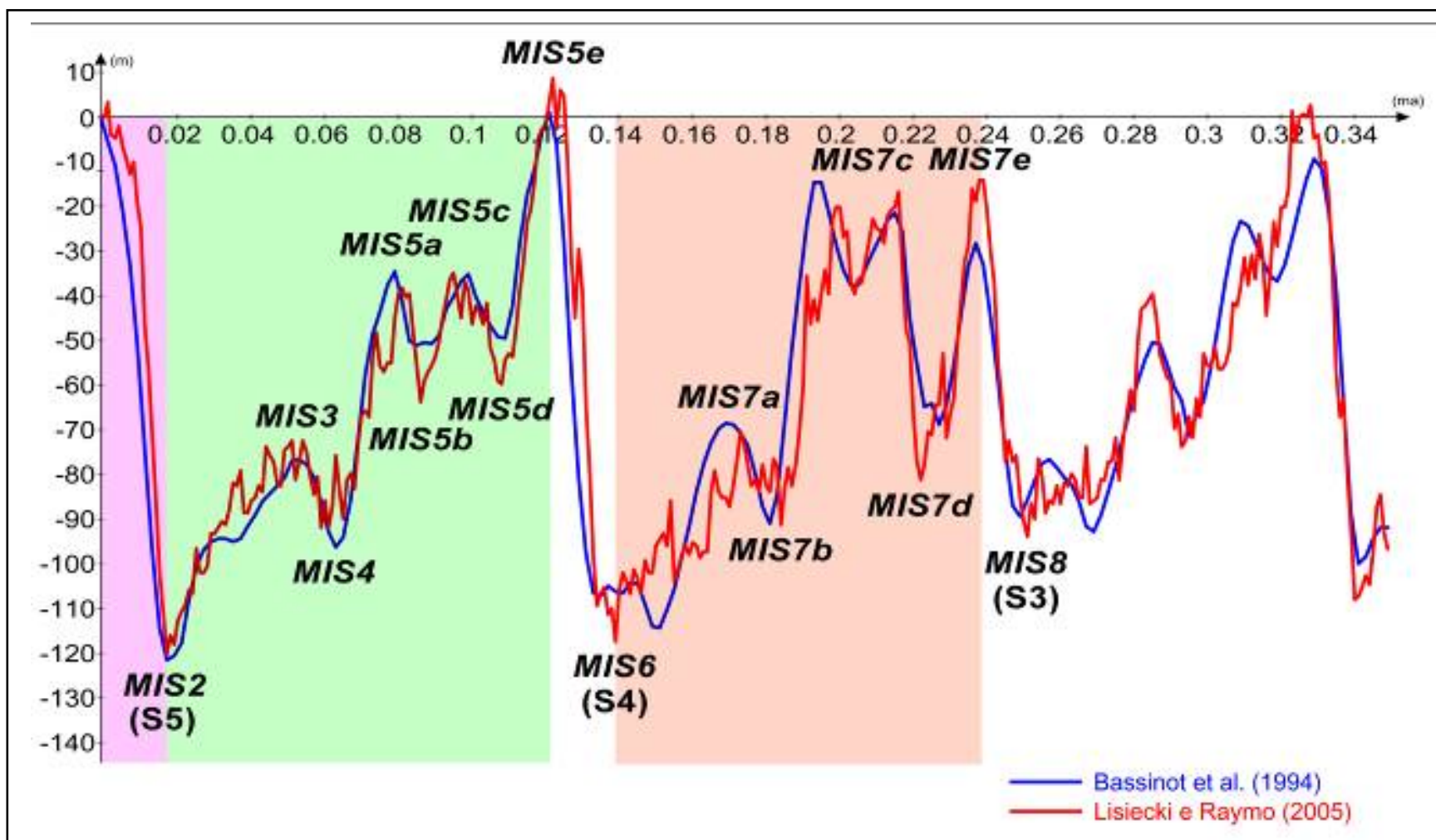

Figura 3: Síntese de dados de nível de mar global a partir de curvas isotópicas de Oxigênio $\delta 18$ propostas por Bassinot et al., (1994) e Lisiecki e Raymo (2005).

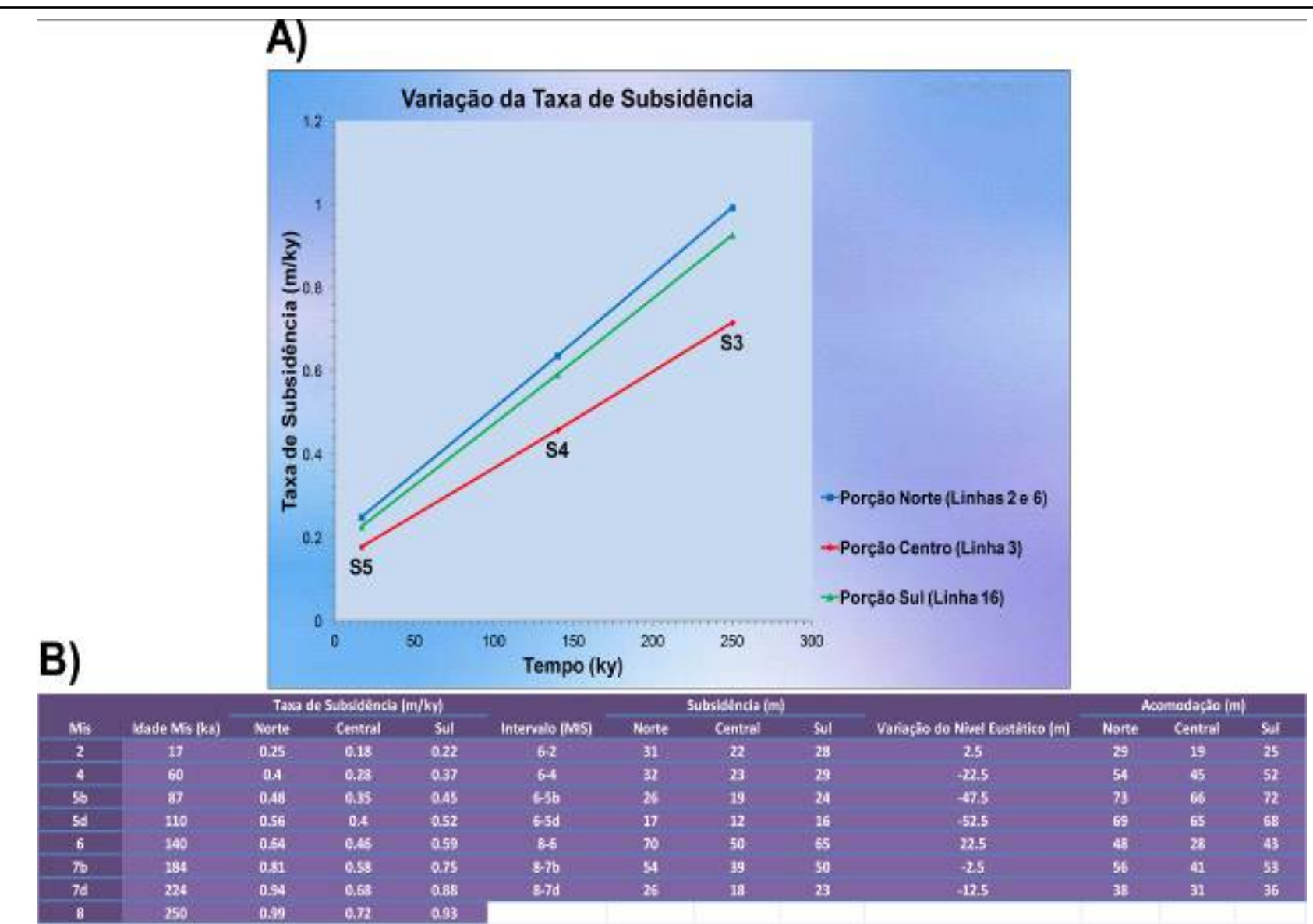

Figura 4: A) Curva de taxa de variação de subsidência no período de 250 ky. B) Tabela com as medidas estimadas de taxa de subsidência, subsidência, variação do nível eustático (MIS) e acomodação para os períodos de formação de cada subunidade sísmica. 